\title{
Modalités d'écriture collaborative et didactique ludique : Les applications pédagogiques des « 24 heures du roman » dans les programmes du Français Langue Seconde
}

\section{Caroline LEBREC, Université de Toronto à Mississauga}

Les « 24 heures du roman » est un projet d'écriture créative et collaborative conçu et réalisé par Anne Forrest-Wilson, avec l'aide de l'association torontoise «L'Écriture en mouvement » 1. Le projet s'est déroulé du 20 au 24 octobre 2015, venant couronner la fin des festivités consacrées au quatre-centenaire de la présence francophone en Ontario. De cet événement est sorti un livre Sur les traces de Champlain : un voyage extraordinaire en 24 tableaux ${ }^{2}$, un documentaire ${ }^{3}$ et du matériel pédagogique ${ }^{4}$. Le projet consistait à réunir un groupe de 24 auteur-e-s et de leur faire explorer l'histoire de la présence francophone en Ontario sur les traces de Samuel de Champlain, avant de la mettre en récit selon vingt-quatre voix différentes, dans un roman constitué de vingtquatre chapitres, lui-même écrit en vingt-quatre heures. Un vrai défi de convergence que ce projet réunissant une grande diversité franco-canadienne, avec pour objectif la mise en avant de l'ensemble dans le respect des voix de chacun.

À partir de la problématique des modalités d'écriture collaborative, cette étude propose dans un premier temps d'analyser les modalités d'écriture collaborative qui permettent à chacune des voix de s'inscrire dans un mouvement entre le collectif (le «nous ») et l'individuel (le « je »). Dans un second temps, les modalités d'écriture collaborative sont appliquées au domaine de la didactique ludique, pour laquelle cette étude fait l'objet d'applications pédagogiques dans les programmes du Francais Langue Seconde (FLS), axées sur l'apprentissage experiential à partir de travaux de groupe mélangeant des étudiants des niveaux A1 à B2 du Cadre Européen Commun de Référence (CECR).

\section{Le projet}

\section{Une diversité franco-canadienne}

Les vingt-quatre voix participants au projet nous sont venues d'Acadie avec les auteur-e-s Herménégilde Chiasson et Gracia Couturier, de France avec les auteur-e-s oulipiens Michèle Audin, Frédéric Forte, Paul Fournel, Hervé Le Tellier, Ian Monk et Olivier Salon, de la communauté franco-ontarienne avec les auteur-e-s Jean M. Fahmy, Vittorio Frigerio, Hélène 
Koscielniak, Jean-Claude Laroque, Daniel Marchildon, Marie-Josée Martin, Mireille Messier, Daniel Soha, Denis Sauvé et Danièle Vallée, de la communauté francophone autochtone avec les auteur-e-s Virginia Pésémapéo Bordeleau et Jean Sioui et enfin du Québec avec les auteur-e-s Yara El-Ghadban, Daniel Grenier, Bertrand Laverdure et Rodney Saint-Éloi.

\section{Un projet à contraintes}

Pour rassembler ces vingt-quatre voix dans un ensemble cohérent, la solution d'une écriture à contrainte a été privilégiée afin d'instaurer des règles communes, à partir desquelles les vingtquatre auteurs pouvaient construire leur récit personnel, selon une esthétique d'entrecroisement des récits. À la base du projet, la contrainte temporelle a consisté à faire écrire les vingt-quatre auteurs en même temps. Pour cela, il fallait une durée suffisamment longue pour laisser aux auteurs le temps d'écrire leur chapitre, mais aussi suffisamment courte pour les garder sous la main pendant toute la durée de l'écriture. Dans un deuxième temps, la contrainte spatiale consistait à réunir les auteurs au même endroit afin que l'aventure de cette écriture collaborative sur les traces de Samuel de Champlain, puisse s'acheminer entre Halifax à Toronto, à bord d'un train, gracieusement mis à disposition de « L'Écriture en mouvement » par Viarail, rendant ainsi possible la combinaison des vingt-quatre auteurs écrivant en vingt-quatre heures, ensemble, à bord d'un train. Aux contraintes temporelle et spatiale s'est également ajoutée la contrainte numérique, reliée cette fois-ci à la productivité et au rendement puisque chaque auteur devait écrire 2800 mots (15 000 signes), chacun, en vingt-quatre heures. Inhabituels dans les sciences humaines qui peinent à se situer dans l'ère quantificative, ces deux concepts se sont révélés être un véritable défi pour certains auteurs, à qui il fallait écrire de manière rapide, claire et efficace, afin que tous les textes soient prêts dans un délai raisonnable, constitué par l'heure d'arrivée du train. Enfin, la contrainte de cohérence a tenu le rôle d'archi-contrainte de ce projet, englobant les trois contraintes susmentionnées (spatiale, temporelle et numérique) - chacune avec un enjeu qui lui est propre; ce afin de constituer un roman construit à partir de vingt-quatre chapitres - un par auteur, ayant une continuité de sens entre eux.

\begin{tabular}{|l|l|l|l|}
\hline Archi-contrainte & Contraintes & Enjeux \\
\hline \multirow{3}{*}{ Cohérence } & Temporelle & $\begin{array}{l}\text { Moment } \\
\text { commun }\end{array}$ & Rendement \\
\cline { 2 - 4 } & Spatiale & Lieu commun \\
\cline { 2 - 3 } & Pumoductivité \\
\hline
\end{tabular}

Fig. 1 : Les contraintes et leurs enjeux dans le projet des « 24 heures du roman ». 


\section{Un livre collectif}

Héritage des pratiques d'écriture collaborative

L'écriture collaborative, peu pratiquée dans le domaine des Belles-Lettres, s'inscrit toutefois dans l'héritage de l'esthétique avant-gardiste qui a vastement renouvelé les pratiques d'écriture traditionnelles, notamment avec les collages dada dont le principe repose sur un mélange de voix et de médias. Plus qu' « une question de technique », le collage est d'abord une position esthétique en rupture chez les avant-gardes, tantôt sous la forme d'une vision d'ensemble, notamment dans les effets de «montage » d'un Cendrars, tantôt sous la forme de «l'irrationnel » pour Dada et les Surréalistes, tantôt sous celle d'un « retour du réel dans l'art » pour les cubistes (Béhar 180-181). Pour notre analyse, nous retiendrons ici l'effet de montage qui permet de donner une cohérence à l'ensemble des voix auctoriales constituant le projet des « 24 heures du roman ». Dans les expérimentations collaboratives des avant-gardes, on retrouve également le cadavre exquis surréaliste. Basé sur des modalités différentes, il permet à cinq auteurs d'écrire une même phrase, où chacun est responsable de l'écriture d'un syntagme (nominal, adjectival ou verbal) selon un agencement contraint d'ordre formel ${ }^{5}$. Le cadavre exquis rejoint l'idée du jeu de papier plié qui consiste à dissimuler les syntagmes, avant de découvrir la phrase qui a été écrite et de réagir à ces associations sémantiques. Ces modalités de jeux d'écriture cachée peuvent être rapprochées de l'écriture simultanée des différents chapitres du livre Sur les traces de Champlain, où chaque auteur, guidé par la liste des consignes, écrivait un texte qui allait trouver sa place dans un espace commun en devenir, donc virtuel ${ }^{6}$.

En restant dans les contraintes formelles, mais en s'éloignant des esthétiques avantgardistes, une pratique collaborative contemporaine se retrouve fréquemment à l'Oulipo, l'Ouvroir de Littérature Potentielle, dont six auteur-e-s participaient au projet des « 24 heures du roman » : Michèle Audin, Frédéric Forte, Paul Fournel, Hervé Le Tellier, Ian Monk, Olivier Salon. Les Oulipiens ont pour habitude d'écrire en se donnant toutes sortes de contraintes, des plus simples (logo-rallye, lipogramme, etc.) aux plus complexes (les règles du jeu de go, etc.). La pratique de l'écriture collaborative s'y fait le plus souvent au nom du groupe, comme le montre le livre $C$ 'est un métier d'homme, où abonde tantôt les effets de reprises et tantôt les effets d'annonce, mais où chaque auteur a su personnaliser le texte-souche à partir duquel les textes de chacun ont été dérivés. Les pratiques collaboratives oulipiennes se situent également parfois entre deux auteurs dont une poétique algorithmique de partage orchestre la répartition des voix, comme par exemple dans Éros 
mélancolique, un ouvrage co-signé par Jacques Roubaud et Anne Garréta (voir Lebrec, «Poétique »). Jeux d'écriture au nom du groupe ou de type restreint (à deux), les pratiques oulipiennes oscillent entre une «esthétique de la complicité » et une « esthétique de la complexité »(Le Tellier $65 ; 218)$ sur lesquelles s'est aligné le projet des « 24 heures du roman ». Ainsi, le premier chapitre, signé par l'oulipien Fournel («Une semaine en enfance », Sur les traces), commence par l'enfance de Samuel de Champlain à Brouage, et met l'accent sur la passion de la mer et la culture maritime selon une filiation de type paternel. En écho au chapitre précédent, le deuxième chapitre rédigé par l'auteure québécoise Yara El Ghadban («Moi, la mer ») se concentre sur la voix d'une mer personnifiée, où l'on retrouve des effets de reprise du personage de l'enfant de Brouage, en écho au chapitre précédent de Fournel. Des effets d'annonce se mettent aussi en place entre les chapitres, avec l'évocation du personnage « Mistenapeo » dans le texte d'El Ghadban; personage qui fera l'objet du chapitre cinq rédigé par l'auteure autochtone Virginia Pésémapéo Bordeleau. Le dernier chapitre signé par Herménégilde Chiasson («Entrevue avec Samuel de champlain ») ferme la boucle en rappelant les aspirations maritimes de l'enfant Samuel de Champlain, qui étaient présentes dès l'incipit du roman. Des exemples de la sorte sont des traces des séances de travail en groupe qui ont eu lieu avant l'embarquement, où des listes de consigne et de thèmes ont été distribués aux auteurs, qui ont pu prendre le temps d'ajuster leur place dans l'ensemble, avant de laisser libre cours au déploiement de leur propre voix dans le roman. Ainsi, partagées par tous et garantes à ne pas laisser de place au hasard dans le processus de montage, les contraintes ont permis que se déploie une écriture du mouvement, permettant à chacun de trouver un espace frontière commun entre leur voix d'auteur et la voix du groupe.

L'écriture collaborative : une écriture du mouvement entre le «je » et le «nous»

Selon Frank Wagner, une voix critique efficace et singulière dans le champ des littératures à contraintes, le texte de type hyperformaliste - construit à partir de contraintes formelles, est souvent considéré comme un texte qui se caractérise par son «impersonnalité » ou effacement de la voix de l'auteur, tandis que pour Wagner, c'est justement dans ce genre de textes qu'un « lieu privilégié du pulsionnel » (431-438; 434) ressort là où l'auteur ne s'y attendait souvent pas. Grâce à la distance formelle créée par l'esthétique de l'écriture à contraintes, l'exploration virtuelle de soi (ou d'un thème, ou d'un lieu, etc.) permet en effet de faire ressortir les traits stylistiques d'un auteur, comme une métahopore de la voix auctoriale, une fois que celle-ci s'est installée dans l'esthétique de la contrainte. Les textes des auteurs oulipiens Fournel (chapitre 1) et Monk 
(chapitre 3) en sont un bon exemple. Dans «Une semaine en enfance », on retrouve des caractéristiques propres à l'écriture de Fournel, de type langagier avec la pratique de l'effet de surprise par la réponse inattendue à une question banalisée (Un homme regarde), de type stylistique avec le clin d'oeil à l'enfance de l'auteur à succès de littérature jeunesse ${ }^{7}$, de type thématique avec l'évocation de la gastronomie (Le bel appétit), de type oulipien avec la pratique du texte à démarreur (Perec / OULIPO) et enfin de type collaboratif avec les formes interrogatives comme autant de pistes lancées aux vingt-trois auteurs partenaires du projet des « 24 heures du roman ». Dans «Le rêve d'Ours-qui-pêche », on retrouve également des caractéristiques propres à l'écriture de Monk, de type formel avec le récit bifurquant (ou arborescent) par la formule « ou bien $»^{8}$ et de type idéologique sur la vérité aveugle des humains, métaphore ici de la voix de l'auteur qui se cherche dans l'ensemble des vingt-quatre voix écrivant en simultané; merveilleux défi du projet des vingt-quatre heures du roman. La part de l'intime donc qui métaphorise, en tout cas dans les deux exemples cités, la figure de l'auteur écrivant.

Ainsi, du construit individuel au partage de l'ensemble, l'écriture collaborative a des caractéristiques qui lui sont propres. En effet, « dialoguer n'est pas la même chose que soliloquer » (Shrage 18). La première caractéristique, au niveau formel, est le montage ou les modalités de croisement des différents récits. La seconde, au niveau interpersonnel, marque l'émergence d'une troisième voix, entre le «je » et le «nous », dans cet espace généré par des modalités qui permettent de «créer une expérience de partage [à la différence du partage] d'une expérience » (3). C'est en cela que l'on passe du collectif au collaboratif. La troisième, au niveau de la contrainte, garantit la cohérence de l'ensemble, en fonction du genre choisi, ici le roman dont les vingt-quatre tableaux représentent les vingt-quatre chapitres.

\section{Le mouvement dans la forme}

Le roman est un genre littéraire qui se prête à toutes les expérimentations, toujours à la recherche de ses limites dans un mouvement allant du centre à sa périphérie. Bruno Blanckeman qualifie ceci de «force centrifuge » de la fiction dans le roman. Jan Baetens, spécialiste de littérature à contraintes, reprend cette métaphore de Blanckeman pour établir les caractéristiques du roman à contraintes. Ainsi, selon Baetens, tandis que le rapport entre la fiction et le roman met en place une force centrifuge de l'exploration des limites, le roman à contraintes offre la mise en place d'un mouvement perpetual entre la force centrifuge, plus connue dans l'esthétique contrainte comme l'écriture des possibles, et la force « centripète » de la contrainte, garante de la cohérence 
de l'ensemble. L'écriture à contraintes est ainsi en elle-même une écriture du mouvement entre le «je » et le «nous», au sein de la forme même de son écriture.

\section{Didactique ludique : Les modalités d'écriture collaborative en classe FLS 9}

\section{Contexte}

À l'automne 2015, les Cafés-rencontre du Département d'études langagières (DLS) de l'Université de Toronto à Mississauga ont été mis sous le signe de l'apprentissage expérientiel, dont les objectifs d'apprentissage sont axés sur l'accomplissement de tâches individuelles. En complément des cours suivis par les étudiants, ces cafés-rencontre offrent un environnement convivial propice à la consolidation des apprentissages langagiers. Ils sont ouverts à tout étudiant inscrit dans les programmes de français. Cette ouverture a l'avantage de dynamiser les programmes offerts par la section française du DSL, en permettant un regrouprement des étudiants selon un principe de complémentarité en fonction de leurs niveaux de compétence, soit débutant, soit intermédiaire, soit avancé. Afin de ne pas déséquilibrer l'accomplissement de la tâche et de permettre une expérience vécue sereinement par les étudiants, la constitution des groupes est un des enjeux principaux du travail collaboratif. Dans ce cas, l'écart de niveau restreint a été privilégié afin qu'une complémentarité puisse s'installer dans les groupes. Les étudiants ont donc été regroupé selon les schémas suivants : débutant/intermédiaire ou intermédiaire/avancé, mais nous avons pris soin d'éviter un regroupement de type débutant/avancé. L'anniversaire de la présence francophone en Ontario en a offert le theme, ancré dans le culturel et dans l'histoire des relations franco-ontariennes.

De l'apprentissage culturel au récit historique oral : «Canada 1615 »

Cette séquence pédagogique se déroule en trois séances de cinquante minutes, dont chacune alterne les moments de travail individuel et de travail de groupe, en fonction des objectifs d'aprentissage suivants :

\begin{tabular}{|l|l|}
\hline Objectifs & Apprentissages expérientiels disciplinaires \\
\hline Culturels & \begin{tabular}{l} 
- Le jeu de 7 familles \\
- Le jeu de 6 familles « Canada $1615 »$ \\
\hline - Les origines de la présence francophone en Ontario \\
• Les liens France/Canada \\
• L'anachronisme
\end{tabular} \\
\hline
\end{tabular}



Langagiers
- Vocabulaire spécifique authentique
- Grammaire de texte
- Cohérence des déictiques énonciatifs
- Mots de liaison
- Création d'un récit oral
- Synthèse
- Écriture d'un récit à plusieurs
Au niveau interpersonnel
- Travail de groupe
- Écriture collaborative
Au niveau personnel
- S'ajuster au groupe
- Trouver sa propre voix au sein du groupe
Au niveau des
- Dynamisation du programme
programmes de français
- Opportunités de rencontre hors-classe
- Opportunités de développement de mentorat entre étudiants

Fig. 2 : Un apprentissage expérientiel dans les programmes de FLS.

Une création de l'association torontoise «L'Écriture en mouvement », «Canada 1615 » est un jeu de cartes offrant un support pictural aux découvertes de Samuel de Champlain sur le territoire canadien et un moment d'apprentissage culturel d'un des jeux de cartes les plus pratiqués en France : le jeu de sept familles. Agrémenté de textes bilingues (français-anglais), le jeu est exploitable à tous les niveaux d'apprentissage. Il se présente sous la forme de six familles dont voici les trente-six possibilités de combinaison offertes aux joueurs :

\begin{tabular}{|l|l|l|l|l|l|}
\hline Colons & $\begin{array}{l}\text { Premières } \\
\text { Nations }\end{array}$ & Animaux & Végétaux & $\begin{array}{l}\text { Vie } \\
\text { quotidienne }\end{array}$ & Lieux \\
\hline $\begin{array}{l}\text { Samuel de } \\
\text { Champlain }\end{array}$ & Algonquins & Moufette & Rutabagas & Calumet & Tadoussac \\
\hline $\begin{array}{l}\text { Hélène de } \\
\text { Champlain }\end{array}$ & Cri & Dindon & Tabac & Tipi & Québec \\
\hline Arquebusier & Micmac & Orignal & Tournesol & $\begin{array}{l}\text { Sirop } \\
\text { d'érable }\end{array}$ & Brouage \\
\hline Écclésiastique & $\begin{array}{l}\text { Nation } \\
\text { Huron } \\
\text { Wendat }\end{array}$ & Castor & Tomates & Maison & Halifax \\
\hline Fermiers & Iroquois & Baleine & Canneberges & Pirogue & Lac Simcoe \\
\hline $\begin{array}{l}\text { Coureur des } \\
\text { bois }\end{array}$ & Montagnais & Ours & Maïs & Tomahawk & Hochelaga \\
\hline
\end{tabular}

Fig. 3 : Les familles du jeu de cartes « Canada $1615 »$.

La première partie de la séquence est dédiée à la familiarisation avec les règles du jeu, les cartes et le vocabulaire spécifique qui lui est attribué, afin de mettre en avant les variations du 
français, tel le verbe «piocher » utilisé en France et le verbe «piger » utilisé dans le français canadien.

\section{Activité 1 : Le jeu de 6 familles « Canada 1615 »}

1) Consultez les règles du jeu traditionnel et prenez un temps pour vous les expliquer au sein de votre groupe. Ces règles vous font-elles penser à un équivalent de jeu de cartes canadien ?

2) À vous de jouer en vous familiarisant avec les expressions suivantes : «Bonne pioche »/ «Mauvaise pioche » et en utilisant les formules suivantes :

Formule 1 : Noms communs

«Dans la famille des je voudrais le/la/les

Exemple : Dans la famille de la «Vie quotidienne », je voudrais le «calumet».

Formule 2 : Noms propres

«Dans la famille des je voudrais . Exemple : Dans la famille des «Colons», je voudrais « Samuel de Champlain».

3) En groupe, créez un récit oral à partir de l'histoire de l'arrivée de Samuel de Champlain en Ontario et à partir des informations données par les cartes que vous avez gagnées au cours du jeu.

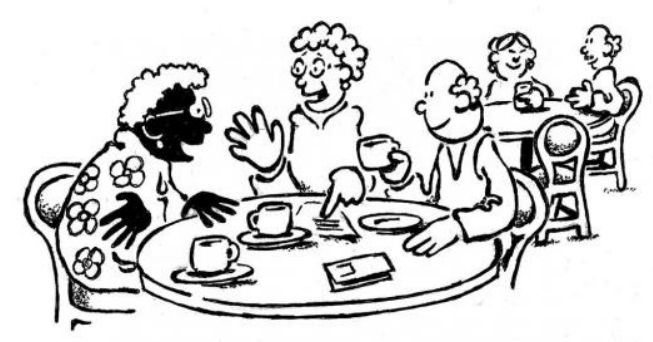

4) Lisez votre récit à tour de rôle.

(Source FLHLMQ).

5) Cherchez des moyens de connecter votre récit aux autres que vous avez entendus.

6) Prolongement : Écrivez un récit commun à partir de tous les récits entendus.

Dans cette première partie de la séquence pédagogique, l'apprentissage expérientiel consiste à mettre en place une situation authentique de jeu qui permet d'aboutir à la création d'un récit oral en groupe. L'aspect ludique est ici renforcé par le choix des cartes qui est un résultat des cartes que le joueur a gagné pendant la partie, au lieu d'être le fruit du hasard (pioche aveugle) ou du choix (pioche découverte).

Du récit historique au récit personnel

La deuxième partie de la séquence consiste à construire la suite d'un récit, en se servant d'éléments biographiques qui permettent de mélanger l'historique et le personnel. 


\section{Activité 2 : Écrivez une suite personnelle à votre récit}

7) Étoffez votre récit : Transcrivez votre récit oral en cherchant à assembler vos phrases à l'aide de mots outils (marqueurs de relation, conjonctions, pronoms relatifs, etc.).

8) Choisissez deux éléments de votre vie contemporaine (voyage, nourriture, etc.) que vous allez pouvoir ajouter à votre récit.

9) Écrivez une première suite à votre histoire, à partir de l'ajout de vos éléments biographiques.

10) Piocher 6 cartes supplémentaires et écrivez une deuxième suite à votre histoire en respectant l'ordre d'apparition des nouvelles cartes dans votre récit et en les mélangeant à votre récit personnel.

11) Lisez les récits obtenus à tour de rôle.

Dans cette deuxième partie de la séquence pédagogique, l'apprentissage expérientiel consiste à introduire du personnel dans l'historique, afin de faire comprendre le concept d'anachronisme. L'aspect ludique est renforcé par l'utilisation des cartes dans leur ordre de pioche.

Du récit historico-personnel au récit collectif

La troisième partie est consacrée à l'acquisition de la pratique d'écriture collaborative.

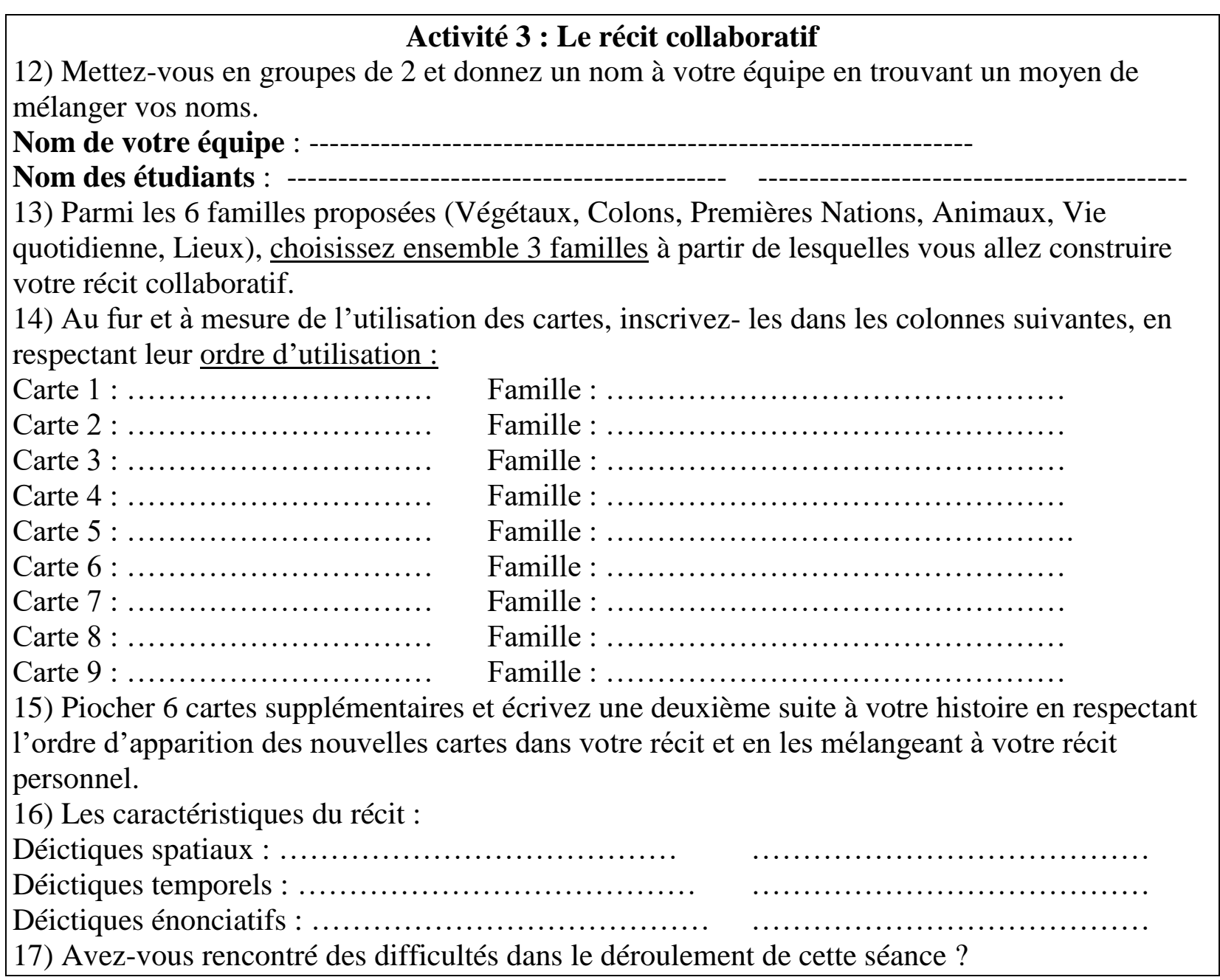


Dans cette troisième et dernière partie de la séquence pédagogique, l'apprentissage expérientiel consiste à présenter les caractéristiques du récit (déictiques et grammaire de texte), à partir desquelles seront déduites celles de l'écriture collaborative, à savoir la présence de la voix de chaque étudiant (le «je»), ainsi que l'harmonisation des voix entre elles (le «nous»). L'aspect ludique est ici caractérisé par la mise en place d'une écriture en mouvement entre les mots utilisés de manière individuelle par un «je » et les mots pouvant réfleter le récit collaboratif du « nous ». L'insertion d'une demande de rétroaction (point 17) consiste à recueillir les réactions des étudiants, probablement mis en situation d'écriture collaborative pour la première fois.

Analyse des résultats

Le groupe dont nous allons commenter les résultats est constitué d'une étudiante de niveau avancé (3e année, série FRE) et une étudiante de niveau intermédiaire (2e année, série FSL) ${ }^{10}$. À l'issue de leur partie, elles ont gagné les 9 cartes suivantes :

(9) Famille des Animaux : orignal, castor, baleine

(9) Famille des Lieux: Québec, Brouage, Lac Simcoe

(9) Famille des Premières Nations: Wendat, Algonquins, Cri

À l'étape de mise en commun de leur texte (Activité 3), le résultat est le suivant, dans lequel nous avons souligné les mots correspondants aux cartes à utiliser :

\section{Texte collaboratif 1}

Samuel de Champlain est allé avec les Hurons Wendat, les Algonquins et les Cris au Québec. Làbas, il a vu un orignal. ( $3^{\mathrm{e}}$ année, FRE)

Le soir, il est resté à Brouage quand il a mangé du castor pour dîner. ( $2^{\mathrm{e}}$ année, FSL) Après avoir mangé le castor, il est allé au lac Simcoe où il a été mangé par une baleine dans laquelle il a trouvé un dindon et ils sont devenus amis. (3e année, FRE)

Les réussites se rerouvent sous la forme du respect des déictiques énonciatifs : Samuel de Champlain/il ; Samuel de Champlain + dindon/ils ; de l'utilisation des mots de liaison : Là-bas ; Le soir ; Après; de 1'emploi des temps du passé : passé composé (est allé x2; a vu; est resté ; $a$ mangé ; a trouvé ; sont devenus), infinitif passé (avoir mangé) et voix passive (a été mangé).

\section{Rétroaction pédagogique 1}

Bien que trop succinct, ce premier texte collaboratif porte les marques d'un récit enchaîné, présentant de solides acquis au niveau de la cohérence des déictiques (énonciatifs, spatiaux et temporels) et de l'agencement syntaxique, en plus de présenter de bon acquis orthographiques et une belle variété de temps verbaux. Seule, la conjonction de subordination temporelle « quand »a été confondu avec le pronom relatif de lieu « où ». Toutefois des formules répétitives sont à noter 
(voir ; manger ; le castor). Les neuf cartes ont bien toutes été utilisées dans leur ordre d'apparition, comme le montre les mots soulignés dans le texte, mais il faut remarquer qu'elles ont seulement été nommées, au lieu d'être exploitées iconographiquement, alors que cela aurait été une stratégie efficace pour étoffer le texte. La carte «Samuel de Champlain », non présentes parmi les cartes gagnées, a été ajoutée comme point de départ du récit, alors que l'on s'attendait à un récit qui se déploie à partir du point de vue des protagonistes appartenant aux Premières Nations, de la personnification des animaux ou d'une description des lieux. Même chose pour l'ajout de la carte « dindon » qui ne figurait pas dans les cartes gagnées. Enfin, si l'alternance des voix et la cohérence ont bien été traitées, on ne peut pas en dire autant de la répartition des tâches : par exemple, l'étudiante de niveau avancé a utilisé sept cartes sur neuf, tandis que l'étudiante de niveau intermédiaire, qui aurait eu besoin d'un plus grand nombre de cartes sur lequel s'appuyer pour développer son récit, n'en a utilisé que deux. À cette étape et pour se préparer à une deuxième tentative, il est donc recommandé à ces étudiantes de varier le lexique, de développer le contenu iconographique des cartes et d'essayer le point de vue des Premières Nations comme nouveau démarreur du récit.

\section{Texte collaboratif 2}

Il était une fois un groupe d'Algonquins qui sont allés au Québec pour chasser des castors. (3e, FRE)

Mais ils n'ont pas trouvé des castors, ils ont trouvé des orignaux. Alors, ils ont décidé d'aller au Brouage pour chercher des castors qu'ils veulent. Là-bas, ils sont rencontré un autre groupe de Huron Wendat qui étaient en train de manger des dindons avec le groupe de $\underline{\text { Cri. }}$. (2e, FSL)

Finalement, le groupe des Algonque a décidé de partir au lac Simcoe pour trouver des castors mais ils ont trouvé des baleines. Pas de chance pour les Algonquins! (3e, FRE)

\section{Rétroaction pédagogique 2}

Les améliorations suggérées dans la rétroaction pédagogique 1 ont été intégrées avec succès. En effet, la répartition des cartes s'est mieux déroulée et le texte est un peu plus développé. De plus, le point de vue des Algonquins a été choisi, ce qui était important pour varier les points de vue sur la présence francophone en Ontario. Toutefois, le texte développé laisse apparaître des difficultés dans la compétence langagière :

(9) Prépositions :

○ $\mathrm{Si}$ «Québec » est ambiguë en français entre le choix de la ville et la région, la traduction anglaise de la carte indiquait «Quebec city ». L'étudiante de niveau avancée aurait donc pu et dû déduire qu'elle devait choisir la préposition «à » (+ ville) et non « au » (+ province). 
○ L'étudiante de niveau intermédiaire n'a pas su identifier Brouage comme une ville ou ne maîtrise pas encore la règle des prépositions devant les villes, à acquérir au niveau A1 selon le cadre européen commun de référence, donc normalement déjà acquis pour le cours dans lequel elle est inscrite.

(9) Groupe nominal :

○ L'article partitif doit être modifié dans une formule négative («ils n’ont pas trouvé de castors »), ce qui est en cours d'acquisition pour le niveau de cette étudiante.

○ «Le groupe de Cri » pose problème, mais si l'étudiante devrait savoir que les noms propres ne portent pas la marque du pluriel en français, en revanche, la règle de l'accord sujet/verbe dans le cas d'un sujet singulier à valeur pluriel sera vu en détail à un niveau plus avancé.

- L'étudiante de niveau avancée manque la dérivation du nom Algonquins, alors que cela est une des premières acquisitions de la langue. Une maîtrise du vocabulaire spécifique à l'activité est toutefois en cours d'acquisition.

(9) Groupe verbal :

- En cours d'acquisition, la concordance des temps n'est pas respectée dans «qu'ils veulent» dans le texte de l'étudiante de niveau intermédiaire, surtout que l'expression nécessite une pensée de l'abstraction marquée par la nécessité du mode conditionnel, qui n'est pas encore maîtrisée à ce niveau. Remarquons toutefois que la forme irrégulière au présent du verbe « vouloir » est quant à elle une réussite, ce qui est censé être maîtrisé à ce niveau.

○ En cours d'acquisition pour l'étudiante de niveau intermédiaire, le choix de l'auxiliaire ÊTRE/AVOIR + CD aux temps composés pose encore problème dans « ils sont rencontré ».

Suite à cette étude, nous pouvons avancer que les modalités de l'écriture collaborative en classe FLS consistent à : être flexible et à l'écoute de son interlocuteur, à être engagé et présent dans la situation de communication, à prendre part à un travail de groupe, à être prêt à assumer une responsabilité partagée dans les tâches accomplies, à écrire un récit cohérent à l'aide de stratégies langagières d'harmonisation des voix et de cohérences syntaxique (déictiques, concordance des temps, etc.) et dans ce cas spécifique, à une participation fictive à l'histoire du Canada francophone et aux relations France/Canada. 


\section{Bibliographie}

Baetens, Jan. Romans à contraintes. Amsterdam : Rodopi, 2005.

Béhar, Henri. Littéruptures. Paris : L’Age d'homme, coll. « Bibliothèque Mélusine », 1988.

Blanckeman, Bruno. Le roman français d'aujourd'hui : transformations, perceptions, mythologies.

Dir. Bruno Blanckeman et Jean-Christophe Millois. Paris : Prétexte, coll. «Critique », 2004.

Burnet, Phillipe, réal. Sur les traces de Champlain : un voyage extraordinaire en 24 tableaux. Machine Gum Productions, 2016. Unis.TV. 2016. Web. Consulté le 29 juin 2016.

Forrest-Wilson, Anne, dir. Sur les traces de Champlain : un voyage extraordinaire en 24 tableaux. Sudbury : Prise de parole, 2015.

Fournel, Paul. Un homme regarde une femme. Paris : Seuil, coll. « Cadre rouge », 1999.

---. Le bel appétit. Paris : P.O.L., 2015.

Frigerio, Vittorio. «Les 24 heures du roman ». Liaison 169 (automne 2015) : 42-43.

Klein Lataud, Christine. «Soyez sur les traces de Champlain ». Liaison 172 (été 2016). Web. Consulté le 29 juin 2016.

L’Écriture en mouvement. «Canada $1615 »$, jeu de 6 familles, 2015.

Lebrec, Caroline. « Contraintes formelles et jeux d'écriture : le cadavre exquis surréaliste et la nine oulipienne ». Mélusine/Formules. Dir. Henri Béhar et Alain Chevrier. Paris-Louvain : Noésis, 2007. 29-42.

---. «Une poétique algorithmique du partage dans Éros mélancolique de Jacques Roubaud et Anne Garréta ». Mélusine XXXII, « À belles mains : Livre surréaliste - livre d'artiste », Dir. Andréa Oberhuber. Paris : L’Âge d'homme, 2012. 177-187.

Le Tellier, Hervé. Esthétique de l'Oulipo. Bordeaux : Castor astral, 2006.

Moore Howard, Rebecca. «Collaborative Learning and Writing ». Writing Matters. McGraw-Hill. Web. Consulté le 29 juin 2016.

Oulipo. C'est un métier d'homme : autoportraits d'hommes et de femmes au repos. Paris : Mille et une nuits, 2010.

Perec, Georges / OULIPO. Le voyage d'hiver et ses suites. Paris : Seuil, coll. « La librairie du XXI siècle », 2013 (1979).

Rue, Émilienne. «Anne Forrest-Wilson (dir.), Sur les traces de Champlain : un voyage extraordinaire en 24 tableaux». Voix plurielles 13.1 (2016). Web. Consulté le 29 juin 2016. 
Shrage, Michael, «Writing to Collaborate: Collaborating to Write », Author-ity and Textuality. Current Views of Collaborative Writing. Dir. James S. Leonard. West Cornwall : Locust Hill, 1994.17-27.

Speck, Bruce W. et al., Collaborative Writing : An Annotated Bibliography. Westport, CT : Greenwood, 1999.

Wagner, Frank. «Le sujet sous contrainte(s)». Le roman français au tournant du XXI ${ }^{e}$ siècle. Dir. Bruno Blanckeman, Aline Mura-Brunel et Marc Dambre, Paris : P de la Sorbonne Nouvelle, 2004. 431-438

\section{Notes}

${ }^{1}$ Un compte rendu de l'événement est disponible dans la revue Liaison : voir Vittorio Frigerio.

${ }^{2}$ Deux comptes rendus de l'ouvrage sont disponibles en ligne : voir Emilienne Rue ; et Christine Klein Lataud.

${ }^{3}$ Sur les traces de Champlain : un voyage extraordinaire en 24 tableaux, Réal. Philippe Burnet. Le documentaire a été diffusé en exclusivité sur Unis.TV le 5 juin à vingt heures. Il est maintenant visible à partir du site Unis.TV ou de $L$ 'Écriture en mouvement à la rubrique consacrée aux « 24 heures du roman ».

${ }^{4}$ Jeu de six familles. Le jeu de cartes peut se commander à partir du site internet de L'Écriture en mouvement, à la rubrique « Ateliers d'écriture ».

${ }^{5}$ Les syntagmes sont réparties selon la formule suivante : Auteur 1/Substantif Sujet + Auteur 2/Adjectif Sujet + Auteur 3/Verbe + Auteur 4/Substantif Objet + Auteur 5/Adjectif Objet. Exemple : « Le cadavre exquis boira le vin nouveau ». Pour les détails de l'analyse concernant le rapport entre le cadavre exquis et la contrainte formelle, voir Caroline Lebrec, «Contraintes formelles ».

${ }^{6}$ Nous utilisons le terme virtuel au sens du potentiel, que lui prête l'Oulipo, l'Ouvroir de Littérature Potentielle. «POTENTIEL, POTENTIELLE adj. (lat. potens, potentis, puissant) Qui existe en puissance, virtuellement : énergie potentielle. Ling. Se dit d'un mode qui indique la possibilité d'une action. Énergie potentielle (Phys.), énergie possédée par un corps, par un système physique, du fait de sa position, de son état » (Le Tellier 22).

${ }^{7}$ Paul Fournel est un auteur jeunesse à succès qui a notamment reçu le Prix du meilleur livre jeunesse en 1982 pour Les aventures très douces de Timothée le rêveur (Paris: Hachette Jeunesse 1978), mais aussi le Prix Renaudot des Lycéens pour Foraine (Paris : Seuil, coll. « Cadre rouge », 1999), le Prix Goncourt de la nouvelle et Grand Prix de la littérature sportive pour Les athlètes dans leur tête en 1989 (Paris : Seuil, coll. « Points », 1988).

${ }^{8}$ Ian Monk est un auteur de littérature populaire et de poésie formelle. Il a publié, entre autres Stoned at Bourges (Les Mille univers, 2007) ; Plouk Town (Paris : Cambourakis, 2007) ; Là (Paris : Cambourakis, 2014).

${ }^{9}$ Pour une bibliographie dans le domaine de l'écriture collaborative en contexte didactique, voir Rebecca Moore Howard «Collaborative Learning and Writing ». Pour une bibliographie plus vaste dans le domaine de l'écriture collaborative, voir Bruce W. Speck et al.

${ }^{10}$ La section FRE regroupe les étudiants inscrits dans des programmes de mineurs, de majeurs et de spécialistes, alliant la langue au culturel dans un contexte littéraire ou linguistique. Les cours de la section FLS offre une mineure en Français aux étudiants inscrits dans d'autres programmes sur le campus de l'Université de Toronto à Mississauga. 